\title{
RETRACEABLE SETS AND RECURSIVE PERMUTATIONS
}

T. G. MCLAUGHLIN

1. By a recursion property of sets of natural numbers, we mean a property preserved under all recursive permutations of the natural numbers; while by a weak-recursion property we mean one which is preserved under recursive equivalence (see [1]). It is known [1] that regressiveness is a recursion property and even a weak-recursion property. We shall prove here that the property of being retraceable fails, by contrast, to be a recursion property, even for sets with recursively enumerable complement. (It is known from [1] that retraceability is not a weak-recursion property.) Our basic terminology and notation are as in [1].

We shall repeat here, for the reader's convenience, the definition of retraceability and regressiveness of sets of natural numbers. An infinite set $\alpha$ of natural numbers is regressive $\Leftrightarrow$ there is a nonrepetitive ordering $a_{0}, a_{1}, a_{2}, \cdots$ of the elements of $\alpha$, and a partial recursive function $p$ whose domain includes $\alpha$, such that $p\left(a_{0}\right)=a_{0}$ and $(\forall n)\left(p\left(a_{n+1}\right)=a_{n}\right) . \alpha$ is called retraceable provided it is regressive with respect to the ordering of its elements in increasing order of magnitude.

2. It is known [1, Proposition 10] that any recursively enumerable set with regressive complement is isomorphic to a recursively enumerable set whose complement is retraceable ("isomorphic to" meaning, of course, the image of-under a recursive permutation); hence, to establish the result claimed in $\$ 1$, it suffices to exhibit a recursively enumerable set whose complement is regressive but not retraceable. This we shall now do.

TheOREM. There exists a recursively enumerable set $\beta$ such that $\beta^{\prime}$, while regressive, is nonretraceable.

Proof. Let $\left\{p_{n}(x)\right\}$ be the usual effective enumeration of the partial recursive functions of one variable, and let " $P_{n}$ " denote the cumulative outcome of performing the first $n$ steps in some fixed effective procedure $P$ for generating precisely all correct equations $p_{q}(k)=r$. With each function $p_{n}$ we associate a marker $\Lambda_{n}$. The set $\beta$, together with a recursive regressing function $f$ for $\beta^{\prime}$, is constructed by stages as follows.

At Stage 0. Place $(2,1),(1,3),(3,0)$, and $(0,0)$ in $f$, attach $\Lambda_{0}$ to 3 , and proceed to Stage 1.

Received by the editors September 10, 1965. 
At Stage $s, s>0$. Let $\Lambda_{t}$ be the marker of largest index which is attached to a number at the end of Stage $s-1$; we assume, as an inductive hypothesis (it will be easily seen, when the description of Stage $s$ is complete, that the assumption persists from stage $s$ to Stage $s+1)$, that $\Lambda_{0}$ is one of the attached markers and that, indeed, all of $\Lambda_{0}, \Lambda_{1}, \cdots, \Lambda_{t}$ are attached at the end of Stage $s-1$. Now examine $P_{s}$. If $P_{s}$ discloses that $p_{0}(3)=2$ and if 2 has not previously been placed in $\beta$, then (a) place 2 in $\beta$, (b) erase all markers $\Lambda_{j}$ for $t \geqq j>0$, (c) place in $\beta$ all numbers $m, m-1, m-2$ such that $m$ was the position of a marker erased in (b), (d) attach $\Lambda_{1}$ to $n+2$, where $n$ is the smallest number not previously placed in the domain of $f$, (e) place $(n+2,1),(n+1, n)$, and $(n, n+2)$ in $f$, and, finally, (f) proceed to Stage $s+1$. Otherwise: if $t \geqq 1$, check to see if $P_{s}$ discloses that $p_{1}(k)=k-1$ where $k$ is the number to which $\Lambda_{1}$ is attached and $k-1$ has not previously been placed in $\beta$. If so, then (a) place $k-1$ in $\beta$, (b) erase all markers $\Lambda_{j}$ for $t \geqq j>1$, (c) place in $\beta$ all numbers $q$, $q-1, q-2$ such that $q$ was the position of a marker erased in (b), (d) attach $\Lambda_{2}$ to $m+2$, where $m$ is the smallest number not previously placed in the domain of $f$, (e) place $(m+2, k-2),(m+1, m),(m, m+2)$ in $f$, and, finally (f) proceed to Stage $s+1$. But otherwise, iterate the foregoing procedure until either (i) some alteration of marker positions and membership in $\beta$ has been made and we have been ordered to go to Stage $s+1$, or (ii) all the positions of $\Lambda_{0}, \cdots, \Lambda_{t}$ at the end of stage $s-1$ have been scrutinized, with no such alterations having been authorized. In case (ii), let $u$ be the least number not yet assigned to the domain of $f$; attach $\Lambda_{t+1}$ to $u+2$, and place in $f$ the pairs $(u+1, u)$, $(u, u+2)$, and $\left(u+2, l^{*}\right)$, where $l^{*}$ is either one or two less than the position $h$ of $\Lambda_{t}$ according as the number one less than $h$ has not or has been placed in $\beta$; then go to Stage $s+1$.

This completes the description of the construction; clearly $\beta$ (= the set of all $n$ placed in $\beta$ at some stage $s \geqq 0$ ) is recursively enumerable.

Remark. At the end of Stage $s$, exactly the numbers $0,1,2, \cdots$, $3(s+1)$ have been assigned to the domain of $f$.

PROOF. Trivial, by induction on $s$. The proof of the theorem is completed by a sequence of three straightforward lemmas.

Lemma A. Every marker $\Lambda_{j}, j \geqq 0$, eventually becomes permanently attached to some one number $n_{j}$, and $n_{j}$ is of the form $3 k, k>0$.

Proof. This is obtained by a routine induction on $j$. (In particular, we must have $n_{0}=3$.)

LEMмA B. $\beta^{\prime}$ is regressed by $f$. 
Proof. We first note that (as is clear from the construction and Lemma A), $\beta^{\prime}$ consists of the numbers $0, n_{j}$ (see Lemma A), $n_{j}-2$, and, provided $p_{j}\left(n_{j}\right) \neq n_{j}-1$, also $n_{j}-1$. From the description of Stage 0 we see that $f(0)=0, f\left(n_{0}\right)=f(3)=0, f\left(n_{0}-2\right)=f(1)=3$, and $f\left(n_{0}-1\right)$ $=f(2)=1$. From the description of Stage $s$, we further have, for each $j>0$, that $f\left(n_{j}-2\right)=n_{j}, f\left(n_{j}-1\right)=n_{j}-2$, and $f\left(n_{j}\right)=n_{j-1}-2$ or $n_{j-1}-1$ according as $n_{j-1}-1$ is or is not in $\beta$. Thus, $f$ does indeed regress $\beta^{\prime}$.

\section{LEMMA C. $\beta^{\prime}$ is not retraceable.}

Proof. Suppose that $p_{k}$ retraces $\beta^{\prime}$. Let $s$ be a stage such that all $\Lambda_{t}, t \leqq k$, are in their final positions prior to $s$, all numbers $\leqq$ the position $n_{k}$ of $\Lambda_{k}$ which are destined for membership in $\beta$ have already gone into $\beta$, and $P_{s}$ discloses that $p_{k}\left(n_{k}\right)=r=$ the next smaller member of $\beta^{\prime}$. If $r=n_{k}-1$, then $n_{k}-1$ must be placed in $\beta$ at or before Stage $s$ : contradiction. Hence $r=n_{k}-2$. But this is possible only if $n_{k}-1$ has previously been placed in $\beta$ owing to discovery of $p_{k}\left(n_{k}\right)=n_{k}-1$ : contradiction. Lemma $C$ follows, and with it the theorem.

3. Remarks. (a) By careful consideration of the proof of Proposition 5 in [1] as it applies to the sets constructed in Theorem T5 of [2], one sees how to show, without a "priority" construction as in $\$ 2$, that retraceability is not always preserved by recursive permutations. The retraceable and regressive sets thus considered do not have recursively enumerable complements (by T4 of [2] and Proposition 10 of [1]); it does follow, however, that there are $2^{N_{0}}$ nonhyperimmune retraceable sets whose retraceability is not preserved by all recursive permutations.

(b) A slight modification in the proof of the theorem leads to the result that primitive recursive permutations need not always preserve retraceability of complements of r.e. sets.

(c) We do not know yet exactly which r.e. degrees contain r.e. sets with regressive, nonretraceable complements; but we can show that any r.e. degree $a$ for which $a^{\prime}=0^{\prime \prime}$ does contain such a set.

\section{REFERENCES}

1. J. C. E. Dekker, Infinite series of isols, Proc. Sympos. Pure Math., Vol. 5, pp. 77-96, Amer. Math. Soc., Providence, R. I., 1962.

2. J. C. E. Dekker and J. R. Myhill, Retraceable sets, Canad. J. Math. 10 (1958), 357-373.

UNIVERSITY OF ILLINOIS 